\title{
A Study of Operators on Fuzzy Sets
}

\author{
Alhaji Jibril Alkali ${ }^{1}$, Sylvanus Kupongoh Samaila ${ }^{2, *}$ \\ ${ }^{1}$ Department of Mathematics, Ahmadu Bello University Zaria, Zaria, Nigeria \\ ${ }^{2}$ Department of Mathematics and Statistics, Federal University Otuoke, Otuoke, Nigeria
}

Email address:

Alkali4real2012@gmail.com (A. J. Alkali),ssylva50@gmail.com (S. K. Samaila)

${ }^{*}$ Corresponding author

\section{To cite this article:}

Alhaji Jibril Alkali, Sylvanus Kupongoh Samaila. A Study of Operators on Fuzzy Sets. Mathematics Letters. Vol. 7, No. 2, 2021 , pp. $30-36$. doi: $10.11648 /$ j.ml.20210702.13

Received: April 4, 2021; Accepted: May 20, 2021; Published: June 15, 2021

\begin{abstract}
Overtime, mathematics had been used as a tool in modeling real life phenomenon. In some cases, these problems cannot fit-into the classical deterministic or stochastic modeling techniques, perhaps due system complexity arising from lack of complete knowledge about the phenomenon or some uncertainty. The uncertainty could either be due to lack of clear boundaries in the description of the object or perhaps due to randomness. In this article, we study a mathematical tool discovered in 1965 by Zadeh suitable for modeling real life phenomenon and examined operations on such a tool. Motivated by the work of Zadeh, we studied operators on Type-1 Fuzzy Sets (T1FSs) and Type-2 Fuzzy sets (T2FSs) and provided examples, one of which is a variant of the Yager complement function for which the complement operator was graphically illustrated. The joint and the meet operators were also studied and examples provided. Non-standard operators were defined on T1FSs and T2FSs and also classified into two groups; the triangular-norm (t-norm) and triangular-conorm (t-conorm). Using tnorm and t-conorm, an example was adopted from Castillo and Aguilar to illustrate the computation of the standard operation on T2FSs. Finally, future research direction was provided based on what is yet to be achieved in fuzzy set theory.
\end{abstract}

Keywords: Fuzzy Sets, Complement Operators, Union Operators, Intersection Operators, T-norm, T-conorm, T1FSs, T2FSs

\section{Introduction}

Overtime, mathematics had been used as a tool in modeling real life phenomenon. In some cases, these problems cannot fit-into the classical deterministic or stochastic modeling techniques, perhaps due system complexity arising from lack of complete knowledge about the phenomenon or some uncertainty. The uncertainty could either be due to lack of clear boundaries in the description of the object or perhaps due to randomness. However, this is not a disadvantage but a blessing in disguise as a mathematical tool capable of accommodating the uncertainty was introduce in 1965 [18] and named fuzzy sets. Interestingly, for over five decades, mathematicians have studied fuzzy sets and found it useful in real life applications as evident in $[2,5,6$, 9, 17, 19-21] and the references therein.

Furthermore, in the study of Zadeh [18] the standard operations on fuzzy sets were first defined. These were the union operations, intersection and complement operations on fuzzy sets, a unique concept in fuzzy set theory without its replica in the Cantorian theory. It has been established in the study of Chen and Zadeh [4] that operations on fuzzy sets has no counterpart in the classical set theory in particular and in classical mathematics in general. Mathematician have extended operations on T1FSs to T2FSs as noticed in [10-12, $14,15]$ and recently $[3,8]$ and the references therein. This fact necessitated the need for further research on the operations on type- 1 and type- 2 fuzzy sets, hence the subject of the present paper.

In addition, as in the classical sense, operators are define on collection of objects with vague boundaries. In this paper, we shall classified operators on fuzzy sets into to two, namely; the triangular-norm operators and the triangularconorm operators defined on type- 1 and type- 2 fuzzy sets. The remaining part of the paper is organised as follows; the next section concerns the preliminaries materials and methods needed in section 3 where we discuss the operators on type-1 fuzzy sets with examples and in section 4 we extend our examples to type-2 fuzzy sets. Finally, in section 5 , we give our concluding note and possible direction of 
future study.

\section{Preliminaries}

Let $A$ be a nonempty set (Cantorian) in a universe $X$. Then, $A$ can be described or characterised via a function called the characteristics or indicator or membership or discrimination function $\chi_{A}: X \rightarrow\{0,1\}$ defined by

$$
\chi_{A}(x)=\left\{\begin{array}{c}
1, \text { if } x \in A \\
0, \text { otherwise }
\end{array}\right.
$$

Evidently, (1) does not accommodate a continuum of membership as an element is either in the set or not. However, this shortfall led to the introduction of fuzzy set theory [18]. Hence, we give the following definition.

Definition [19]: Let $X$ be a universe of discourse, a fuzzy set $\tilde{A}$ in $X$ is a set of ordered pairs

$$
\tilde{A}=\left\{\left(x, \mu_{\tilde{A}}(x) \mid x \in X\right)\right\} .
$$

Where $\mu_{\tilde{A}}(x): X \rightarrow[0,1]$ is the membership function of the generic element mapping all the elements in the universe of discourse into the membership space $[0,1]$ Therefore, the membership in a fuzzy set is a continuum of gradation. However, the fuzzy set so defined is a Type-1 Fuzzy Set since the membership values are real numbers. We shall denote type-1 fuzzy sets as T1FSs Now, let $\mu: X \rightarrow[0,1]$ be a fuzzy subset of a nonempty universe $X$, we shall denote the totality of all such fuzzy sets in $X$ by $\mathcal{F}(\mathrm{x})$. In particular, if the universe of discourse is the set of real numbers $\mathbb{R}$, then the gradation membership $\mu: \mathbb{R} \rightarrow[0,1]$ is called a fuzzy number, which is obviously an extension of the classical set of real number system and we denote the set of all fuzzy numbers by $\mathcal{R}_{\mathcal{F}}$.

Definition [1]: Let $\mathcal{R}_{\mathcal{F}}$ be the set of all fuzzy subsets of $\mu: \mathbb{R} \rightarrow[0,1]$ satisfying the following conditions;

1. $\forall \mu \in \mathcal{R}_{\mathcal{F}}, \mu$ is upper semi-continuous

2. $\forall \mu \in \mathcal{R}_{\mathcal{F}}, \mu$ is fuzzy convex

3. $\forall \mu \in \mathcal{R}_{\mathcal{F}}, \mu$ is normal

4. $\overline{\{x \in \mathbb{R} \mid \mu(x)>0\}}$ is compact.

Then $\mathcal{R}_{\mathcal{F}}$ is called the space of fuzzy numbers. Clearly, $\mathbb{R} \subset \mathcal{R}_{\mathcal{F}}$.

Definition [2]: Let $\tilde{A}$ be a fuzzy subset of some universe of discourse $X$ and $\alpha \in[0,1]$. Then, the set

$$
\tilde{A}_{\alpha}=\{x \mid x \in X, \tilde{A}(x) \geq \alpha\}
$$

is called the $\alpha$-level set or the $\alpha$-cut and the set

$$
\operatorname{supp} \tilde{A}=\{x \in X \mid \tilde{A}(x)>0\}
$$

is the support of the level set. The core of the level set is given by

$$
\tilde{A}_{1}=\{x \in X \mid \tilde{A}(x)=1\} .
$$

Now, from (1), we have the following representation theorem.

Theorem 2.1 [2] Let $\tilde{A}$ be a fuzzy set in some universe $X$. for any element $x \in X$, its membership degree to $\tilde{A}$ can be expressed as follows

$$
\tilde{A}(x)=\bigcup_{x \in \tilde{A}_{\alpha}} \alpha .
$$

Definition: A membership function $\mu$ is said to be upper semi-continuous if and only if the $\alpha$-level sets (1) are closed.

Definition: A Membership function $\mu$ is said to be lower semi-continuous if and only if the set

$$
\{x \in X: \mu(x)>\alpha\}, \alpha \in[0,1] \text { is open }
$$

\section{Operators on Type-1 Fuzzy Sets}

In this section, standard and non-standard operators on T1FSs and their examples are presented. To this end, let $\tilde{A}, \widetilde{B}, \tilde{C}$ and $\widetilde{D} \in \mathcal{F}(\mathrm{X})$ be T1FSs, then, [18] defines the standard operators on T1FSs as follows;

$\mu_{\tilde{A}^{c}}(x)=1-\mu_{\tilde{A}}(x)$ the cmpement operator

$\mu_{\tilde{C}}(x)=\mu_{\tilde{A} \cup \tilde{B}}(x)=\max \left\{\mu_{\tilde{A}}(x), \mu_{\tilde{B}}(x)\right\}$ the joint operator

$\mu_{\widetilde{D}}(x)=\mu_{\tilde{A} \cap \tilde{B}}(x)=\min \left\{\mu_{\tilde{A}}(x), \mu_{\tilde{B}}(x)\right\}$ the meet operator

Obviously, operations on T1FSs are some kind of generalisations of their counterpart in the classical set theory. Thus, the above operators will form the basis for defining non-standard operators. Therefore, in the following result, the properties of these operators are characterised.

Proposition 3.1 [7]. Considering the basic connectives in fuzzy set theory, the following properties hold;

1. $\left(\tilde{A}^{c}\right)^{c}=\tilde{A}$. Involution

$$
\text { 2. } \tilde{A} \cup \tilde{B}=\tilde{B} \cup \tilde{A}
$$

$\tilde{A} \cap \tilde{B}=\tilde{B} \cap \tilde{A}$. Commutativity

$$
\text { 3. }(\tilde{A} \cup \tilde{B}) \cup \tilde{C}=\tilde{A} \cup(\tilde{B} \cup \tilde{C})
$$

$(\tilde{A} \cap \tilde{B}) \cap \tilde{C}=\tilde{A} \cap(\tilde{B} \cap \tilde{C})$. Associativity

$$
\text { 4. } \tilde{A} \cap(\tilde{B} \cup \tilde{C})=(\tilde{A} \cap \tilde{B}) \cup(\tilde{A} \cap \tilde{C})
$$

$\tilde{A} \cup(\tilde{B} \cap \tilde{C})=(\tilde{A} \cup \tilde{B}) \cap(\tilde{A} \cup \tilde{C})$. Distributive

$$
\text { 5. } \tilde{A} \cup \tilde{A}=\tilde{A}
$$

$\tilde{A} \cap \tilde{A}=\tilde{A}$. Idempotency

$$
\text { 6. } \tilde{A} \cup(\tilde{A} \cap \tilde{B})=\tilde{A}
$$

$$
\tilde{A} \cap(\tilde{A} \cup \tilde{B})=\tilde{A} \text { Absorption by } \tilde{A}
$$

$$
\tilde{A} \cup X=X
$$

$\tilde{A} \cap \phi=\phi$. Absorption by $X$ and $\phi$

$$
\text { 7. } \tilde{A} \cup \phi=\tilde{A}
$$

$$
\tilde{A} \cap X=\tilde{A} \text {. Identity }
$$

$$
\text { 8. } \overline{\tilde{A} \cup \tilde{B}}=\overline{\tilde{A}} \cap \overline{\tilde{B}}
$$

$\overline{\tilde{A} \cap \tilde{B}}=\overline{\tilde{A}} \cup \overline{\tilde{B}} \cdot$ De Morgan's law 

[6].

Proof. The proof of the above proposition can be found in

Remark 3.2 We remark that the law of contradiction and the law of excluded middle does not hold in fuzzy sets.

Proposition 3.3 [2]. If $\tilde{A}$ is a non-classical fuzzy set $\tilde{A}: X \rightarrow[0,1]$. Then,

(i) $\tilde{A} \cap \tilde{A}^{c} \neq \phi$

(ii) $\tilde{A} \cup \tilde{A}^{c} \neq X$

Proof. see [2]

\subsection{The Complement Operator}

Let us take the universe of discourse $X=[0,1]$. Then, an operator $C:[0,1] \rightarrow[0,1]$ is called the complement operator and it mapped the membership function $\mu_{\tilde{A}}(x)$ of a fuzzy set $\tilde{A}$ to $[0,1]$ and the mapped value is denoted by $C\left(\mu_{\tilde{A}}(x)\right)$ and satisfies the following complement axioms; Axiom-C1 $C(0)=1, C(1)=0$ Boundary condition

Axiom-C2 for any $\mu_{\tilde{A}}(x), \mu_{\tilde{A}}(y), x, y \in X$. If $\mu_{\tilde{A}}(x)<$ $\mu_{\tilde{A}}(y)$, then $C\left(\mu_{\tilde{A}}(x)\right) \geq C\left(\mu_{\tilde{A}}(y)\right)$ Monotonicity Condition

Aaxiom-C3 $C$ is a continuous operator

Axiom-C4 $C$ is involutive.

Remark 3.4 It is sufficient for a complement function to satisfy axiom-C1 and axiom-C2 known as the "axiomatic skeleton" [13]

Example 3.1 [13] The function

$$
C(a)=\left\{\begin{array}{l}
1, \text { if } \mathrm{a} \leq \mathrm{t} \\
0, \text { if } \mathrm{a}>t
\end{array}\right.
$$

The function satisfy only the "axiomatic skeleton" conditions and it is a complement function. Exampe 3.2. The function $C(x)=1-\sin \left(\frac{\pi x}{2}\right), x \in[0,1]$ is a complement function that satisfy axioms-C1-C3 but not axiom-C4.

Verification.

It is sufficient to verify that axiom-C4 does not hold. Now, let $x=0.23 \in[0,1]$, then $C(0.23)=0.65$ but $C(0.65)=$ 0.15 and since $0.23 \neq 0.15$ the involution property fails. Exampe 3.3 The function

$$
C(x)=\left\{\begin{aligned}
1, \text { if } \mathrm{x}=0 \\
1-\mathrm{x}, \text { if } 0<x \leq 1
\end{aligned}\right.
$$

is a complement function that satisfy all the four axioms (axiom-C1-axiom-C4).

Solution

It suffices to show the involutive property. To this end, let $0.1 \in(0,1]$, then $C(0.1)=0.9$ and $C(0.9)=0.1$ showing that the involution property hold.

Exampe 3.4 [13]. The Yager function is given as

$$
C_{w}(a)=\left(1-a^{w}\right)^{\frac{1}{w}} \text { where } w \in(-1, \infty) .
$$

This function reduces to the standard complement function for $w=1$.

Exampe 3.5. This example is a variant of the Yager Complement function defined as

$$
C(x)=\left\{\begin{array}{c}
1, \text { if } \mathrm{x}=0 \\
\sqrt{(1-\mathrm{x})}, \text { if } 0<x \leq 1
\end{array}\right.
$$

This function satisfy axiom-C1, axiom-C2 and axiom-C3 but not axiom-C4.

\subsection{Fuzzy Union Operator}

An operator $U$ is called the union operator provided the following axioms hold; Axiom-U1: $U(0,0)=0, U(0,1)=$ $1, U(1,0)=1, U(1,1)=1$ Boundary Condition

Axiom-U2: $\quad U(x, y)=U(y, x) \quad, \quad$ for any $x, y \in$ $[0,1]$. Commutativity property

Axiom-U3: If $x \leq x^{\prime}$ and $y \leq y^{\prime}$, then $U(x, y) \leq U\left(x^{\prime}, y^{\prime}\right)$ Axiom-U4: For any $x, y, z \in[0,1], \quad U(U(x, y), z)=$ $U(x, U(y, z))$ Associativity Property.

In the study [13] axiom-U1 to axiom-U4 are called the "axiomatic skeleton" and the necessary axioms that a function must satisfy to be a fuzzy union operator. In what follows, two more operators are added

Axiom-U5: $\mathrm{U}$ is continuous

Axiom-U6: $\mathrm{U}$ is idempotent. That is $U(x, x)=x$

Next we provide some example to demonstrate the operator so define.

Exampe 3.6. Consider the fuzzy sets defined as

$$
\tilde{A}(x)=\left\{\begin{array}{c}
0, \text { if }<1 \\
\frac{\mathrm{x}-1}{6}, \text { if } 1 \leq \mathrm{x}<7 \\
10-\mathrm{x}, \text { if } 7 \leq \mathrm{x}<10 \\
0, \text { if } 10<x
\end{array}\right.
$$

and

$$
\tilde{B}(x)=\left\{\begin{array}{c}
0, \text { if } \mathrm{x}<2 \\
\mathrm{x}-2, \text { if } 2 \leq \mathrm{x}<3 \\
1, \text { if } \mathrm{x} \leq \mathrm{x}<4 \\
\frac{6-\mathrm{x}}{2,} \text { if } 4 \leq \mathrm{x} \leq 6 \\
0, \text { if } 6<x
\end{array}\right.
$$

Therefore, the joint of the two fuzzy sets is

$$
\begin{gathered}
\tilde{A} \cup \tilde{B}=\mu_{\tilde{A} \cup \tilde{B}}(x)=\max \left\{\mu_{\tilde{A}}(x), \mu_{\tilde{B}}(x)\right\} \\
\tilde{A} \cup \tilde{B}=\{(0,0),(1,0),(2,0.17),(3,1),(4,1),(5,0.67), \\
(6,0.83),(7,1),(8,0.67),(9,0.33),(10,0)\} .
\end{gathered}
$$

Example 3.7 Let $X$ denote the universe of the hight (in $\mathrm{cm}$ ) of students in Ahmadu Bello University,. Zaria. Then, $X=\{140,150,160,170,180,190\}$. Consider the fuzzy sets "Short", "Average hight" and "tall". Our interest is to use the Yager's union operator using these sets. to this end, Let

$$
\tilde{A}(x)
$$$$
=\{(140,1),(150,1),(160,0.9),(170,0.7),(180,0.3),(190,0)\} \text {, }
$$$$
\tilde{B}(x)
$$$$
=\{(140,0),(150,0),(160,0.1),(170,1),(180,0.8),(190,0)\}
$$

and

$$
\tilde{C}(x)
$$


$=\{(140,0),(150,0),(160,0),(170,0),(180,0.3),(190,1)\}$.

The Yager's union operator satisfy axioms-U1-U5 but not idempotency. The function is defined as

$$
U_{w}(a, b)=\min \left\{1,\left(a^{w}+b^{w}\right)^{\frac{1}{w}}\right\}, w \in(0, \infty)
$$

For $w=1$, the Yager function reduces to $U_{1}(a, b)=$ $\min \{1, a+b\}$, for $w=2$, the Yager function becomes $U_{2}(a, b)=\min \left\{1, \sqrt{\left(a^{2}+b^{2}\right)}\right\}$. However, as $w \rightarrow \infty$, the function becomes

$$
U_{\infty}(a, b)=\lim _{w \rightarrow \infty} \min \left\{1,\left(a^{w}+b^{w}\right)^{\frac{1}{w}}\right\}=\max \{a, b\} . \text { where }
$$
$\mathrm{a}$ and $\mathrm{b}$ are membership fucntions

Now, we shall use the example on hight of students considering "short" and "Average hight" as our fuzzy sets. This is illustrated in table 1 for $w=1$, table 2 for $w=2$ and table 3 for $w=\infty$.

Table 1. The Yager Union Operator for $w=1$.

\begin{tabular}{lllllll}
\hline $\boldsymbol{a}, \boldsymbol{b}$ & $\mathbf{1}$ & $\mathbf{1}$ & $\mathbf{0 . 9}$ & $\mathbf{0 . 7}$ & $\mathbf{0 . 3}$ & $\mathbf{0}$ \\
\hline 00 & 1 & 1 & 0.9 & 0.7 & 0.3 & 0 \\
00 & 1 & 1 & 0.9 & 0.7 & 0.3 & 0 \\
00.1 & 1 & 1 & 1 & 0.8 & 0.4 & 0.1 \\
11.0 & 1 & 1 & 1 & 1 & 1 & 1 \\
00.8 & 1 & 1 & 1 & 1 & 1 & 1 \\
00 & 1 & 1 & 0.9 & 0.7 & 0.3 & 0 \\
\hline
\end{tabular}

Table 2. The Yager Union Operator for $w=2$.

\begin{tabular}{lllllll}
\hline $\boldsymbol{a}, \boldsymbol{b}$ & $\mathbf{1}$ & $\mathbf{1}$ & $\mathbf{0 . 9}$ & $\mathbf{0 . 7}$ & $\mathbf{0 . 3}$ & $\mathbf{0}$ \\
\hline 0 & 1 & 1 & 0.9 & 0.7 & 0.3 & 0 \\
0 & 1 & 1 & 0.9 & 0.7 & 0.3 & 0 \\
0.1 & 1 & 1 & 1 & 0.8 & 0.4 & 0.1 \\
1.0 & 1 & 1 & 1 & 1 & 1 & 1 \\
0.8 & 1 & 1 & 1 & 1 & 1 & 0.8 \\
& 1 & 1 & 0.9 & 0.7 & 0.3 & 0 \\
\hline
\end{tabular}

Table 3. The Yager Union Operator for $w=\infty$.

\begin{tabular}{lllllll}
\hline $\boldsymbol{a}, \boldsymbol{b}$ & $\mathbf{1}$ & $\mathbf{1}$ & $\mathbf{0 . 9}$ & $\mathbf{0 . 7}$ & $\mathbf{0 . 3}$ & $\mathbf{0}$ \\
\hline & 1 & 1 & 0.9 & 0.7 & 0.3 & 0 \\
& 1 & 1 & 0.9 & 0.7 & 0.3 & 0 \\
.1 & 1 & 1 & 0.9 & 0.7 & 0.3 & 0.1 \\
.0 & 1 & 1 & 1 & 1 & 1 & 1 \\
.8 & 1 & 1 & 0.9 & 0.8 & 0.8 & 0.8 \\
& 1 & 1 & 0.9 & 0.7 & 0.3 & 0 \\
\hline
\end{tabular}

Exampe 3.8. The Probabilistic Sum Operator: The Fuzzy probabilistic sum $\tilde{A} \widehat{+} \tilde{B}$ is defined as

$$
\mu_{\tilde{A} \tilde{+} \tilde{B}}(x)=\mu_{\tilde{A}}(x)+\mu_{\tilde{B}}(x)-\mu_{\tilde{A}}(x) \mu_{\tilde{B}}(x) \forall x \in X .
$$

It has been established in [13] that the probabilistic sum satisfy commutative, associative, identity and De Morgan's law. Revisiting our previous example on fuzzy sets modeling the linguistic "Short" and "Average hight" we obtained the probabilistic sum as

$$
\mu_{\tilde{A} \tilde{+} \tilde{B}}(x)=
$$

$\{(140,1),(150,1),(160,0.91),(170,1),(180,0.86),(190,0)\}, \forall x$
$\in X$

Exampe 3.9. The Bounded Sum [13]: The bounded sum
$\tilde{A} \oplus \tilde{B}$ is defined as

$$
\mu_{\tilde{A} \oplus \tilde{B}}(x)=\min \left\{1, \mu_{\tilde{A}}(x)+\mu_{\tilde{B}}(x)\right\} \forall x \in X .
$$

Clearly, the Yager fuzzy union function reduces to the bounded operator for $w=1$, hence, table 1 is the illustration.

Exampe 3.10. Drastic sum [13]: The Drastic sum $\tilde{A} \uplus \tilde{B}$ is defined as

$$
\mu_{\tilde{A} \uplus \tilde{B}}(x)=\left\{\begin{array}{c}
\mu_{\tilde{A}}(x), \text { if } \mu_{\tilde{B}}(x)=0 \\
\mu_{\tilde{B}}(x), \text { if } \mu_{\tilde{A}}(x)=0 \\
1, \text { for others }
\end{array}\right.
$$

Exampe 3.11. Hamacher's Sum [13]: The Hamacher's sum $\tilde{A} \cup \tilde{B}$ is define as

$$
\begin{gathered}
\mu_{\tilde{A} \cup \tilde{B}}(x)=\frac{\mu_{\tilde{A}}(x)+\mu_{\tilde{B}}(x)-(2-\gamma) \mu_{\tilde{A}}(x) \mu_{\tilde{B}}(x)}{1-(1-\gamma) \mu_{\tilde{A}}(x) \mu_{\tilde{B}}(x)} \\
\forall x \in X, \gamma \geq 0
\end{gathered}
$$

Again, using are previous example we obtained the Hamacher's Sum which is also a fuzzy set as

$$
\begin{gathered}
\mu_{\tilde{A} \cup \tilde{B}}(x) \\
=\{(140,1),(150,1),(160,0.92),(170,1),(180,0.91),(190,0)\}
\end{gathered}
$$

\subsection{Fuzzy Intersection Operator}

Let $I:[0,1] \times[0,1] \rightarrow[0,1]$ be an operator. Then $I$ is called a fuzzy intersection operator defined as

$$
\mu_{\tilde{A} \cap \tilde{B}}(x)=I\left[\mu_{\tilde{A}}(x), \mu_{\tilde{B}}(x)\right]=\min \left\{\mu_{\tilde{A}}(x), \mu_{\tilde{B}}(x)\right\}
$$

provided $I$ satisfy the following axioms

Axiom-I1: Boundary Condition

$$
I[0,0]=0, I[0,1]=0, I[1,0]=0, I[1,1]=1
$$

Axiom-I2: Commutativity

$$
I(a, b)=I(b, a) \text { for } \mu_{\tilde{A}}(x)=a \text { and } \mu_{\tilde{B}}(x)=b .
$$

Axiom-I3: Monotonic nondecreasing

If $a \leq a^{\prime}, b \leq b^{\prime}$, then $I(a, b) \leq I\left(a^{\prime}, b^{\prime}\right)$

Axiom-I4: Associativity

$$
I(I(a, b), c)=I(a, I(b, c))
$$

The four axioms are called the "axiomatic skeleton". However, two more axioms are added

Axiom-I5: I is a continuous function

Axiom-I6: Idempotency

$$
I(a, a)=a
$$

The Following are examples of the fuzzy intersection functions.

Exampe 3.12. The Yager fuzzy intersection function. This function is defined as

$$
\begin{gathered}
I_{w}(a, b)=1-\min \left\{1,\left((1-a)^{w}+(1-b)^{w}\right)^{\frac{1}{w}}\right\}, w \in \\
(0, \infty) .
\end{gathered}
$$

This function reduces to $I_{1}(a, b)=1-\min \{1,2-a-$ 
b\}, for $w=1$

and $I_{2}(a, b)=1-\min \left\{1-\sqrt{(1-a)^{2}+(1-b)^{2}}\right\}$, for $w=2$. However, for very large $w$, the function becomes

$$
\begin{aligned}
I_{\infty}(a, b)=\lim _{w \rightarrow \infty}[1- & \left.\min \left\{1,\left((1-a)^{w}+(1-b)^{w}\right)^{\frac{1}{w}}\right\}\right] \\
= & \min (a, b) .
\end{aligned}
$$

Exampe 3.13. The Algebraic Product. The algebraic product $\tilde{A} \dot{\widetilde{B}}$ is defined as

$$
\mu_{\tilde{A} \dot{B}}(x)=\mu_{\tilde{A}}(x) \dot{\tilde{B}}(x) \forall x \in X .
$$

Exampe3.14. Bounded Product. The bounded product $\tilde{A} \odot \quad \tilde{B}$ is defined as

$$
\mu_{\tilde{A} \odot \tilde{B}}(x)=\max \left\{0, \mu_{\tilde{A}}(x)+\mu_{\tilde{B}}(x)-1\right\} \forall x \in X
$$

\subsection{Classifications of Operators on T1FSs}

This section is devoted to classifications of operators on T1FSs. Thus, the operators are classified into two groups namely; the triangular-norm operators and the triangularconorm operators.

Definition [13] A binary operation $*:[o, 1] \times[0,1] \rightarrow[0,1]$ is a triangular-norm (t-norm) provided the following conditions holds

(i) $\quad x * y=y * x \quad$ for $\quad(x, y) \in[0,1] \times[0,1]$. Commutativity property

(ii) $\quad x *(y * z)=(x * y) * z \quad$ for $\quad x, y, z \in[0,1]$. Associativity property

(iii) If $x \leq y$, then $x . z \leq y \cdot z$ monotonicity property

(iv) $x * 1=1$. The boundary condition

Therefore, following the above definition, the following operators are t-norms

1. The Fuzzy Intersection Operator

2. The Algebraic Product Operator

3. The Bounded Product Operator and

4. The Drastic Product Operator.

Definition $3.5[13]$ Let $T:[0,1] \times[0,1] \rightarrow[0,1]$ be an operator. Then, $\forall x, y, x^{\prime}, y^{\prime}, z \in[0,1] \mathrm{T}$ is said to be a triangular-conorm (t-conorm) provided the following conditions holds

(i) $T(x, 0)=0, T(x, 1)=1$. Boundary Condition

(ii) $T(x, y)=T(y, x)$. Commutativity

(iii) If $\left(x \leq x^{\prime}, y \leq y^{\prime}\right) \rightarrow T(x, y) \leq T\left(x^{\prime}, y^{\prime}\right)$. Monotonicity

(iv) $T(T(x, y), z)=T(x, T(y, z))$. Associativity.

Therefore, the fuzzy union operator, the algebraic sum operator, the bounded sum operator, the Drastic sum operator and the Disjoint sum operators ars t-conorm operators.

\section{Operators on Type-2 Fuzzy Sets (T2FSs)}

In this section, we denote Type-2 Fuzzy Sets (T2FSs) by $\hat{A}$ and is devoted to operations on T2FSs. Thus, we begin with a definition
Definition (Type-2 Fuzzy Sets). A fuzzy set $\hat{A}$ define in some universe of discourse $X$, having primary membership $u$ say and secondary membership $\mu_{\hat{A}}(x)$ is called a T2FS, if the secondary membership function is a T1FS. That is $\hat{A}: X \rightarrow$ $\mathcal{F}([0,1])$ defined as

$$
\begin{gathered}
\hat{A}=\int_{x \in X} \mu_{\hat{A}}(x) / x=\int_{X}\left[\int_{J_{x}^{u}} f_{x}(u) / u / x, J_{x}^{u}=\{(x, u): u \in\right. \\
\left.\left[\underline{\mu}_{\hat{A}}(x), \bar{\mu}_{\hat{A}}(x)\right]\right\} \subseteq[0,1]
\end{gathered}
$$

Consequently, the above definition can be extended to type-3 fuzzy sets and in general, type-n fuzzy sets if the secondary membership functions depend on T2FS and type(n-1) fuzzy sets respectively. However, we conjecture that mathematical theory establishing the fact that T2FSs provides better results at all times than T1FSs remain open so also mathematical theory for type-3 fuzzy sets. Next we present a representation theorem of T2FSs.

Theorem 4.1 [14] Assume that the primary variables $x$ is sample at $N$ values, $x_{1}, x_{2}, \ldots, x_{N}$ and at each of these values is its primary membership $u_{i}$ are sampled at $u_{i}$ values $u_{i_{1}}, u_{i_{2}}, \ldots, u_{i_{m i}}$. Let $\hat{A}_{e}^{j}$ denote the $j^{\text {th }}$ type- 2 embedded set for T2FS Â. That is,

$$
\begin{gathered}
\hat{A}_{e}^{j} \equiv\left\{x_{i},\left(u_{i}^{j}, f_{x_{i}}\left(u_{i}^{i}\right)\right), u_{i}^{j} \in\right. \\
\left.\left\{u_{i_{k}}, k=1, \ldots, M_{i}, i=1, \ldots, N\right\}\right\}
\end{gathered}
$$

in which $f_{x i}\left(u_{i}^{j}\right)$ is the secondary grade at $u_{i}^{j}$. Then, $\hat{A}$ can be represented as the union of its type- 2 embedded sets, i.e

$$
\hat{A}=\sum_{i=1}^{n_{A}} \hat{A}_{e}^{j}, n_{A}=\prod_{i=1}^{N} M_{i} .
$$

However, let us consider two T2FSs

$$
\begin{gathered}
\hat{A}=\int_{x \in X} \mu_{\hat{A}}(x) / x=\int_{X}\left[\int_{J_{x}^{u}} f_{x}(u) / u\right] / x, J_{x}^{u}=\{(x, u): u \in \\
\left.\left[\mu_{\hat{A}}(x), \bar{\mu}_{\hat{A}}(x)\right]\right\} \subseteq[0,1]
\end{gathered}
$$

and

$$
\begin{gathered}
\hat{B}=\int_{x \in X} \mu_{\hat{B}}(x) / x=\int_{X}\left[\int_{J_{x}^{w}} f_{x}(w) / w\right] / x, J_{x}^{w}= \\
\left\{(x, w): w \in\left[\underline{\mu}_{\hat{B}}(x), \bar{\mu}_{\hat{B}}(x)\right]\right\} \subseteq[0,1] .
\end{gathered}
$$

Therefore, following the argument in [15], that the union of two T2FSs is another T2FSs we defined the union of T2FSs.

Definition [3] Let $\hat{A}$ and $\hat{B}$ be any two T2FSs. Then, the union of $\hat{A}$ and $\hat{B}$ is given as $\hat{A} \cup \hat{B}=\int_{x \in X}\left[\mu_{\hat{A}}(x) \cup\right.$ $\left.\mu_{\hat{B}}(x)\right] / x=\int_{u \in J_{x}^{u}} \int_{w \in J_{x}^{w}} f_{x}(u) \star g_{x}(w) /(u \cup w) \forall x \in X$.

In computations, the joint between two T2FSs is carried out point-wisely between every pair of primary membership. The union operations on T2FSs is computed as t-conorm operation between the corresponding secondary grades of $\mu_{\hat{A}}(x), \mu_{\hat{B}}(x), f_{x}(u)$ and $g_{x}(w)$ respectively $\forall x \in X$. When more than one combination of any two primary membership gives the same point $u \cup w$, then in the joint we keep the one with the largest membership grade. 
In all, Karnik and Mendel [11] argue that due to the complexity in computing the joint of T2FS for arbitrary $\mathrm{T} 2 \mathrm{FSs}$, that is, for $n$ convex and normal T1FSs $F_{1}, F_{2}, \ldots, F_{n}$ characterised by membership functions $f_{1}(\theta), \ldots, f_{n}(\theta)$, respectively, where $f_{1}\left(v_{1}\right)=\ldots f_{n}\left(v_{n}\right)=1$ and $f_{i}(\theta)$ are reordered so that $v_{1} \leq v_{2} \leq \ldots \leq v_{n}$, the membership function of $U_{i=1}^{n} f_{i}$ using the maximum t-conorm and either minimum or product t-norm can be expressed as

$$
\mu_{\sqcup_{i=1}^{n}} F_{i}(\theta)=\left\{\begin{array}{c}
T_{i=1}^{n} f_{i}(\theta) \text { if } \theta<v_{1} \\
T_{i=1}^{k} f_{i}(\theta), \text { if } v_{k} \leq \theta \leq v_{k+1}, 1 \leq k \leq n-1 \\
\bigcap_{i=1}^{n} f_{i}(\theta)
\end{array}\right.
$$

However, the above formula is di if $\theta>v_{n}$. fficult in computation.

Definition [14]. As in T1FSs, the intersection of any two T2FSs is also a T2FS whose membership function is computed via

$$
\begin{gathered}
\mu_{\hat{A} \cap \hat{B}}(x)=\int_{u \in J_{x}^{u}} \int_{w \in J_{x}^{w}} f_{x}(u) \star \frac{g_{x}(w)}{u \cap v} \\
=\mu_{\hat{A}}(x) \sqcap \mu_{\widehat{B}}(x), \forall x \in X .
\end{gathered}
$$

Where $\Pi$ denote the meet operation. AS in the case of the joint operation, the meet between two secondary membership functions $\mu_{\hat{A}}(x)$ and $\mu_{\hat{B}}(x), v=u \cap w$ must be performed between every possible pair of primary membership $u$ and $w$, such that $u \in J_{x}^{u}$ and $w \in J_{x}^{w}$ and the secondary grade must be computed as the t-norm operation between the corresponding secondary grade for all $x \in X$. If however, more than one combination of any two primary membership gives the same point $u \cap w$, then we keep the one with the largest membership grade. More over, [11] constructed

$$
\mu_{\Pi_{i=1}^{n}} F_{i}(\theta)=\left\{\begin{array}{c}
\bigcup_{i=1}^{n} f_{i}(\theta), \text { if } \theta<v_{1} \\
\bigcap_{i=1}^{k} f_{i}(\theta), \quad \text { if } v_{n} \leq \theta \leq v_{k+1}, 1 \leq \\
\bigcap_{i=1}^{n} f_{i}(\theta), \\
\quad k \leq n-1
\end{array}\right.
$$

as the formula for the membership of $\cap_{i=1}^{n} f_{i}$ using the maximum t-conorm $(U)$ and the minimum t-norm $(n)$. Sadly, the above formula is difficult in application and its variant for the product t-norm of (6) remains of open till date.

Definition. The complement of a T2FS is yet another T2FS and is define as

$$
\hat{A}^{\prime}=\int_{X} \mu_{\hat{A}^{\prime}}(x) / x
$$

Finally, in this section, we provide an example and demonstrate how operations on T2FSs are computed.

Exampe 4.2. [3] Let $\hat{A}$ and $\hat{B}$ be two T2FSs, with membership function given as

$$
\mu_{\widehat{A}}(x)=0.5 / 0.1+0.8 / 0.2 \text { and } \mu_{\widehat{B}}(x)=0.4 / 0.5+0.9 / 0.9
$$

using the operations of t-norm and t-conorm, we have that,

$$
\begin{gathered}
\mu_{\hat{A} \cup \hat{B}}(x)=\mu_{\hat{A}}(x) \cup \mu_{\hat{B}}(x)=(0.5 / 0.1+0.8 / 0.2) \cup(0.4 / 0.5+0.9 / 0.9) \\
=(0.5 \cap 0.4) /(0.1 \cup 0.5)+(0.5 \cap 0.9) /(0.1 \cup 0.9)+(0.8 \cap 0.4) /(0.2 \cup 0.5)+(0.8 \cap 0.9) /(0.2 \cup 0.9) \\
=0.4 / 0.5+0.5 / 0.9+0.4 / 0.5+0.8 / 0.9 \\
=\max \{0.4,0.4\} / 0.5+\max \{0.5,0.8\} / 0.9 \\
=0.4 / 0.5+0.8 / 0.9 \\
\begin{aligned}
\mu_{\hat{A} \cap \hat{B}}(x)=\mu_{\hat{A}}(x) \cap \mu_{\hat{B}}(x)=(0.5 / 0.1+0.8 / 0.2) \cap(0.4 / 0.5+0.9 / 0.9) \\
=(0.5 \cap 0.4) /(0.1 \cap 0.5)+(0.5 \cap 0.9) /(0.1 \cap 0.9)+(0.8 \cap 0.4) /(0.2 \cap 0.5)+(0.8 \cap 0.9) /(0.2 \cap 0.9) \\
=0.4 / 0.1+0.5 / 0.1+0.4 / 0.2+0.8 / 0.2 \\
=\max \{0.4,0.5\} / 0.1+\max \{0.4,0.8\} / 0.2 \\
=0.5 / 0.1+0.8 / 0.2 \\
\mu_{\hat{A}^{\prime}}(x)=0.5 /(1-0.1)+0.8 /(1-0.2) \\
=0.5 / 0.9+0.8 / 0.8
\end{aligned}
\end{gathered}
$$

\section{Conclusion and Future Works}

In this paper, existing literature on operation on T1FSs and T2FSs were reviewed and examples were provided to show the computational aspect of the membership function. However, it is worthy of note to mention that computational aspects of these operators are difficult especially in T2FSs. we Identify some open problems which include among others lack of mathematical theory establishing the fact that T2FS is by far better than T1FSs in modeling linguistic and an establish theory in particular on type-3 fuzzy sets and in general type-n fuzzy sets. We also pointed out that a variant of (7) constructed is yet to be establish. In future research work, we shall aim at looking at the problems. 


\section{References}

[1] Armand, A., Allahviranloo, T. and Gouyandeh G. (2019). Some undamental results on fuzzy calculus. Iranian Journal of Fuzzy Systems 15 (3), 27-46.

[2] Bede, B. (2013). Mathematics of Fuzzy Sets and Fuzzy Logic, Springer-Verlag, New York.

[3] Castillo, O. and Aguilar, T. (2019). Type-2 Fuzzy logic in control of nonsmooth systems: Theoretical concepts and Applications. Springer Nature, Switzerland AG, Switzerland.

[4] Chang S. and Zadeh, L. A. (1972). On Fuzzy Mappings and Control. IEEE Transactions on systems Man and cybernetics, SMC-2 (1) 30-34.

[5] Chen, G. and Pham, T. (2001). Introduction to fuzzy sets, fuzzy logic and fuzzy control systems CRC Press, New York.

[6] Dubois, D. and Prade, H. (1980). Fuzzy stes and systems: Theory and Applications. Academic Press, INC, New York.

[7] Dubois, D. and Prade, H. (2000). Fundamentals of Fuzzy Sets, Kluwer Academic Publishers, New York.

[8] H. Garg,, H., Gwak, J., Mahmood, T. and Ali, Z. (2020). Power Aggregation Operators and VIKOR methods for a complex q-Rung Orthopair Fuzzy Sets and Thier Applications mathematics, 8,538 .

[9] Gen, M., Tsujimura, Y., and Zheng, D. (1997). An application of fuzzy set theory to inventory control model. Computers Ind. Engng 33, 553-556.

[10] Hamrawi, H. (2011). Type-2 fuzzy alpha-cuts PhD Thesis, De Mentfort University, London, (2011).
[11] Karnik, N., and Mendel, J. (2001a). Centroid of a type-2 fuzzy set. Information Sciences 132, 195-220.

[12] Karnik, N. and Mendel, J. (2001b). OPerations on Type-2 Fuzzy sets. Fuzzy Sets and Systems 122, 327-348.

[13] Lee, H. K., (2005). First Course on Fuzzy sets: Theory and Applications Springer.

[14] Mendel, J. M., (2017). Advances in type-2 fuzzy sets and systems Information Sciences 177, 84-110.

[15] Mizumoto, M. and Tanaka, K (1976). Some Properties of fuzzy sets of type-2. Information and Controll.

[16] Mizumoto, M. and Tanaka, K. (1981). Fuzzy sets of type-2 under product and algebraic sum Fuzzy sets and Systems 5, 277-290.

[17] Negoita, C. and Ralescu, D., (1975). Application of Fuzzy sets to systems Analysis Springer Basel AG, Germany.

[18] Zadeh, L. A. 1965. Fuzzy Sets, Information and Control 8, 338-353.

[19] H. J. Zimmermann, H-J (1996). Fuzzy set theory and its Applications 3rd Edition. Kluwer Academic Publishing Group, London.

[20] H. J. Zimmermann, H-J (1991) Fuzzy set theory and its Applications $2^{\text {nd }}$ Edition. Kluwer Academic Publishing Group, USA (1991).

[21] Zimmermann, H-J, (2001). Fuzzy set theory and its Applications 4th Edition. Springer Science + Business Media LLC, New York. 